genommenem Weiterlaufen der $\mathrm{CO}_{2}$-Reduktion zur Erklärung unserer Befunde wohl aus.

Eine solche Annahme würde außerdem sowohl den Befunden $\mathrm{K}$ andlers ${ }^{5}$ bei der Untersuchung des Glucoseeinbaus im Licht in Gegenwart von Hemmstoffen, den Versuchsergebnissen von Winterman s ${ }^{6}$ über eine Anhäufung von Polyphosphaten bei Chlorella im Licht trotz fehlender $\mathrm{CO}_{2}$, als auch neuen Befunden von Bidwell und Mitarbb. ${ }^{39}$, nach denen bei Glucosefütterung im Licht der Zuckerabbau gehemmt wird, widersprechen.

39 R. G. S. Bidwell, G. Krotk ov u. B. Reed, Canad. J. Bot. 33, 189 [1955].
Wir können also bei der oben vorgetragenen Auffassung bleiben, daß die Primärprozesse der Photosynthese selbst im Licht die Energie für die Glucoseaufnahme bzw. für die ersten Schritte des Glucoseeinbaues in die Zelle liefern. Diese Energie steht damit nicht mehr zur Verfügung, so daß es zu der festgestellten Hemmung der $\mathrm{CO}_{2}$-Assimilation kommt.

Für technische Assistenz bei den Versuchen danke ich Fräulein H. L. Wilk ening. Der De uts che n F or $\mathrm{sch} u \mathrm{ng}$ gemeins chaft bin ich für die Förderung der Arbeit durch Personal- und Sachbeihilfen sehr zu Dank verpflichtet.

\title{
NOTIZEN
}

\section{Versuche mit radioaktiver Indolylessigsäure zur Prüfung der sogenannten Ablenkung des Wuchs- hormonstromes durch Licht}

Von Erwin Bünning und $\mathrm{H}$ ans-J. Reisener Botanisches Institut der Universität Tübingen

und Friedrich Weygand, Helmut Simon und Johann Friedrich Klebe

Organisch-chemisches Institut der Technischen Universität Berlin-Charlottenburg

(Z. Naturforschg. 11 b, 363-364 [1956]; eingeg. am 27. März 1956)

Zur Erklärung des Phototropismus der Avena-Koleoptilen (Keimscheiden des Hafers) wird meist mit einer Ablenkung des Wuchshormonstromes durch Licht gerechnet. Wirklich nachgewiesen ist als Komponente dieses Phototropismus bisher wohl eine Zerstörung, nicht aber die Ablenkung des Hormonstromes. Wir wollten durch Versuche mit radioaktiver Indolylessigsäure (IES) das Vorkommen jener Ablenkung prüfen.

Für die Versuche wurde krist. $\beta$-Indolylessigsäure[2-14C] verwendet (Aktivität $2 \mathrm{mC} / \mathrm{mMol}$ ), deren Synthese in dieser Zeitschrift bereits beschrieben wurde 1 .

Als Versuchsmaterial diente Siegeshafer von Svalöf. Von den $2-3 \mathrm{~cm}$ langen Koleoptilen wurde die äußerste Spitze (etwa $0,2 \mathrm{~mm}$ ) mit einer Rasierklinge abgetrennt und auf die so entstandene Schnittfäche, die den Koleoptil-Hohlraum noch nicht freilegte, entweder Wassertropfen (Versuch $1-3$ ) oder ein ungefähr $1 \mathrm{~mm}^{3}$ großer Agarblock mit dem Wuchsstoff aufgesetzt (im erstgenannten Fall verhinderte ein Wollfettring das Herablaufen der Flüssigkeit). Die Koleoptilen wurden $50 \mathrm{~min}$ lang einseitig beleuchtet. Beleuchtet wurde mit Glühbirnen, und zwar entweder mit etwa $40 \mathrm{Lx}$ einer $60 \mathrm{~W} / 220 \mathrm{~V}$ - oder mit etwa $100 \mathrm{Lx}$ einer $30 \mathrm{~W} / 6 \mathrm{~V}$-Birne (in diesem Fall

1 F. W e y g a nd u. H. L ind e n, Z. Naturforschg. 9 b, 682 [1954].

\begin{tabular}{|c|c|c|c|c|c|c|}
\hline \multirow[b]{2}{*}{ Versuch } & \multirow{2}{*}{$\begin{array}{c}\text { IES-Kon- } \\
\text { zentration } \\
{\left[\mathrm{g} / \mathrm{cm}^{3}\right]}\end{array}$} & \multirow{2}{*}{$\begin{array}{c}\text { Anzahl } \\
\text { Koleoptilen }\end{array}$} & \multirow[b]{2}{*}{ Koleoptilflanke } & \multicolumn{3}{|c|}{ Impulse $/ \mathrm{min} / \mathrm{mg}$ Trockengewicht } \\
\hline & & & & FHZ 15 & ZR I & ZR III \\
\hline 1 & $5 \cdot 10^{-6}$ & 100 & Licht & 4,04 & $69,8 \pm 0,9$ & \\
\hline 2 & $5 \cdot 10^{-6}$ & 100 & $\begin{array}{l}\text { Schatten } \\
\text { Licht }\end{array}$ & 4,10 & $\begin{array}{l}78,0 \pm 0,9 \\
407\end{array}$ & $43.5+0.8$ \\
\hline 2 & 5.10 & 100 & Schatten & $\begin{array}{l}1,83 \\
2,4\end{array}$ & $\begin{array}{l}40, \\
35,9 \pm 0,7\end{array}$ & $\begin{array}{l}4,3,5 \pm 0,0 \\
38,7 \pm 0,7\end{array}$ \\
\hline 3 & $5 \cdot 10^{-6}$ & 100 & Licht & 1,12 & $20,2 \pm 0,5$ & \\
\hline 4 & $5 \cdot 10^{-6}$ & 120 & $\begin{array}{l}\text { schatten } \\
\text { Licht }\end{array}$ & $\begin{array}{l}1,20 \\
3,56\end{array}$ & $\begin{array}{l}20,0 \pm 0,5 \\
82,9 \pm 1,0\end{array}$ & $83,0+1,0$ \\
\hline & & & Schatten & 3,57 & $86,8 \pm 1,0$ & $86,7 \pm 1,0$ \\
\hline 5 & $5 \cdot 10^{-7}$ & 120 & $\begin{array}{l}\text { Licht } \\
\text { Schatten }\end{array}$ & $\begin{array}{l}1,16 \\
1,08\end{array}$ & & \\
\hline 6 & $5 \cdot 10^{-7}$ & 72 & Licht & 0,57 & & \\
\hline 7 & $5 \cdot 10^{-8}$ & 450 & $\begin{array}{l}\text { Licht } \\
\text { Schatten }\end{array}$ & & $\begin{array}{l}0,89 \pm 0,07 \\
0,67 \pm 0,04\end{array}$ & $\begin{array}{l}0,87 \pm 0,07 \\
0,74 \pm 0,04\end{array}$ \\
\hline
\end{tabular}

Tab. 1. Aktivität in den belichteten und unbelichteten Längshälften von Avenakoleoptilen, die mit den in Spalte 2 angegebenen Konzentrationen radioaktiver IES behandelt und anschließend 50 min einseitig belichtet wurden. 
paralleles Licht). 60 min nach Beginn der Bestrahlung war die positiv phototropische Krümmung sichtbar, noch 30 min später wurden die Koleoptilen längs halbiert und nach Trocknung die Radioaktivität von Licht- und Schattenseite gesondert bestimmt. Zur Messung diente bei den meisten Proben ein Zählrohr FHZ 15 (Meßgerät von Frieseke \& Hoepfner), bei einigen Proben zusätzlich (in Versuch 7 nur) nach Verbrennung der Proben Gaszählrohre (ZR I oder ZR III der Tabelle) unter Zugabe von nichtradioaktivem Äthan. Die aus Tab. 1 ersichtlichen Werte wurden gefunden (Nulleffekte abgezogen).

Aus der Tab. 1 ist ersichtlich, daß die Aktivitätsdifferenzen von Licht- und Schattenseite, soweit eine Fehlerberechnung möglich war, meist innerhalb der Fehlergrenzen liegen. Außerdem können Differenzen ebensooft zugunsten der Lichtseite wie zugunsten der Schattenseite bestehen.

Da für die Gaszählrohre 100 Impulse/min/mg minus Nulleffekt etwa $6 \cdot 10^{-6} \mathrm{mg}$ IES entsprechen und eine Koleoptile ungefähr $0,5 \mathrm{mg}$ Trockengewicht aufweist, hatten wir bei unseren Versuchen etwa $10^{-11}$ bis $10^{-9} \mathrm{~g}$ radioaktiver IES in einer Koleoptile. Die normale Menge beträgt etwa $1 \cdot 10^{-10} \mathrm{~g}$. Man kann also nicht annehmen, der hypothetische Ablenkungseffekt sei durch eine Überschwemmung mit Wuchsstoff verdeckt worden.

Die Versuche bieten somit keinerlei Stütze für die zur Diskussion gestellte Ablenkung. Wir sollten jene oft vertretene Annahme, obwohl wir sie wegen der Verletzung der Koleoptile nicht endgültig widerlegen können, jetzt als eine Hypothese von recht zweifelhaftem Wert betrachten, also lieber versuchen, den Phototropismus nur durch Phänomene wie Wuchsstoffinaktivierung, -aktivierung usw. zu erklären. Das gleiche möchten wir für den Geotropismus annehmen; auch für diese Reaktion ließ ınser erster Versuch keinen Anhaltspunkt für die Annahme einer Querverschiebung erkennen.

\section{Neue Organo-bor-verbindungen}

Von Ulrich Krüerke

Institut für Anorganische Chemie, München

(Z. Naturforschg. 11 b, 364-365 [1956]; eingeg. am 18. Febr. 1956)

Trotz zahlreicher Arbeiten auf dem Gebiet der organischen Borverbindungen ist bisher nur wenig bekannt über das Verhalten von Borverbindungen mit ungesättigten organischen Resten 1. Acetylenderivate des Bors sind in der Literatur überhaupt noch nicht beschrieben worden. Im Hinblick auf die Elektronenlücke am Boratom beanspruchen diejenigen Substanzen besonderes Interesse, bei welchen eine direkte Beziehung zwischen Boratom und Kohlenstoff-Dreifachbindung besteht, also Verbindungen der Konstitution $>\mathrm{B}-\mathrm{C} \equiv \mathrm{C}-\mathrm{R}$. - Die Darstellung eines Bor-trialkinyls gelang bisher noch nicht; es konnten aber verwandte Verbindungen und verschiedene Zwischenprodukte isoliert werden. Sie bilden den Gegenstand dieser und folgender Mitteilungen.

1 Vgl. T. D. Parson u. D. M. Ritter, J. Amer. chem. Soc. 76, 1710 [1954]; R. L. Le t s ing e r u. I. H. S k o o g, J. org. Chem. 18, 895 [1953]; E. R ot hstein, R. W. S aville, J. chem. Soc. 1952, 2987; u. a.

$$
\begin{gathered}
\text { Natrium-tetra-(phenyläthiny lo })- \\
\text { borat, } \mathrm{Na}\left[\mathrm{B}(-\mathrm{C} \equiv \mathrm{C}-\mathrm{Ph})_{4}\right]
\end{gathered}
$$

Bei Versuchen zur Darstellung eines Bor-tri-phenyläthinyls nach der Gleichung:

$\mathrm{BCl}_{3}+3 \mathrm{Ph}-\mathrm{C} \equiv \mathrm{C}-\mathrm{Na} \rightarrow \mathrm{B}(-\mathrm{C} \equiv \mathrm{C}-\mathrm{Ph})_{3}+3 \mathrm{NaCl}$

beobachtete ich das Auftreten einer stabilen, kristallisierenden Verbindung mit Salzcharakter. Es mußte sich dabei um ein Additionsprodukt von. Phenylacetylen-natrium an primär gebildetes Bor-tri-phenyläthinyl handeln:

$$
\begin{aligned}
\mathrm{B}(-\mathrm{C} \equiv \mathrm{C}-\mathrm{Ph})_{3}+\mathrm{Ph}-\mathrm{C} \equiv & \mathrm{C}-\mathrm{Na} \rightarrow \\
& \begin{array}{l}
\mathrm{Na}\left[\mathrm{B}(-\mathrm{C} \equiv \mathrm{C}-\mathrm{Ph})_{4}\right] \\
(\mathrm{I}) .
\end{array}
\end{aligned}
$$

Mit dieser Deutung stand die Tatsache in Einklang, daß trotz eines nach Gl. (1 a) genau eingehaltenen Molverhältnisses der Komponenten (1:3) nach der Umsetzung nicht substituierte $\mathrm{B}-\mathrm{Cl}$-Bindungen festgestellt wurden (hydrolysierbares Chlor). Dementsprechend stiegen die Ausbeuten an der Komplexverbindung, wenn die Reaktion mit einem Molverhältnis 1:4 (Gl. 1 a und $1 \mathrm{~b}$ ) durchgeführt wurde.

Der normale Ablauf der Umsetzung (1) wird durch Nebenreaktionen sehr gestört: Es bilden sich, offenbar unter der Einwirkung der Bor-Halogen-Bindung, polymere Produkte von tiefroter Farbe, welche die Isolierung der Substanz (I) außerordentlich erschweren und die Ausbeuten nicht über $20 \%$ steigen lassen. $\mathrm{BBr}_{3}$ und $\mathrm{BCl}_{3}$ sind in dieser Hinsicht besonders wirksam, durch Anwendung von $\mathrm{BF}_{3}$ kann das Auftreten gefärbter Produkte vermieden werden. Aus diesem Grunde muß die Umsetzung mit einem Überschuß Phenylacetylen-natrium im Reaktionsmedium durchgeführt werden, so daß sich bei diesem Verfahren die komplexe Bindung des $\mathrm{B}(-\mathrm{C} \equiv \mathrm{C}-\mathrm{Ph})_{3}$ nach $\mathrm{Gl}$. (1 b) nicht umgehen läßt.

Man versetzt also Phenylacetylen-natrium als Suspension in Benzin (Umsetzung mit $\mathrm{BCl}_{3}$ oder $\mathrm{BBr}_{3}$ ) oder in Äther (bei Verwendung von $\mathrm{BF}_{3}$ - Ätherat) unter Rühren langsam mit dem Borhalogenid. Die Reaktion mit $\mathrm{BCl}_{3}$ und $\mathrm{BBr}_{3}$ erfordert Temperaturen um $-60^{\circ}$, wenn nicht weitgehend Polymerisation eintreten soll, während bei der Umsetzung von $\mathrm{BF}_{3}$ auch in siedendem Äther keine gefärbten Nebenprodukte entstehen. Das Salz (I) kann dann aus dem Niederschlag durch Ausziehen mit Tetrahydrofuran oder besser mit Äther isoliert werden.

Natrium-tetra-(phenyläthinylo)-borat (I) löst sich sehr gut in Tetrahydrofuran und kristallisiert aus der konzentrierten Lösung mit $3 \mathrm{Mol}$. Solvens in schön ausgebildeten monoklinen Prismen oder Kristallnadeln (bei schnellem Abkühlen). Das Salz ist farblos. Unter Verlust von 2 Mol. Tetrahydrofuran tritt an der Luft Verwitterung ein. Das dritte Solvens-Mol. ist fester gebunden, wird aber beim Erwärmen auf $80^{\circ}$ im Vakuum schnell abgegeben. Der aus einer Lösung von Tetrahydrofuran durch schnelle Fällung mit Benzin entstehende flockige Niederschlag enthält von vornherein nur 1 Mol. Solvens. Diese Fällungsmethode eignet sich zur Reinigung der Verbindung. Die Wechselwirkung mit Diäthyläther scheint geringer zu sein: es lösen sich nur etwa $6 \mathrm{mMol}$ des Salzes 